\title{
Vitiligo-like Leukoderma as an Indicator of Clinical Response to Immune Checkpoint Inhibitors in Late- stage Melanoma Patients
}

\section{Sofia Verkhovskaia}

IDI IRCCS: Istituto Dermopatico dell'Immacolata Istituto di Ricovero e Cura a Carattere Scientifico

Francesca Romana Di Pietro

IDI IRCCS: Istituto Dermopatico dell'Immacolata Istituto di Ricovero e Cura a Carattere Scientifico

\section{Simona Mastroeni}

IDI IRCCS: Istituto Dermopatico dell'Immacolata Istituto di Ricovero e Cura a Carattere Scientifico

\section{Maria Luigia Carbone}

IDI IRCCS: Istituto Dermopatico dell'Immacolata Istituto di Ricovero e Cura a Carattere Scientifico

\section{Damiano Abeni}

IDI IRCCS: Istituto Dermopatico dell'Immacolata Istituto di Ricovero e Cura a Carattere Scientifico

\section{Roberto Morese}

IDI IRCCS: Istituto Dermopatico dell'Immacolata Istituto di Ricovero e Cura a Carattere Scientifico

Francesca Maria Morelli

IDI IRCCS: Istituto Dermopatico dell'Immacolata Istituto di Ricovero e Cura a Carattere Scientifico

\section{Stefania D’Atri}

IDI IRCCS: Istituto Dermopatico dell'Immacolata Istituto di Ricovero e Cura a Carattere Scientifico

Paolo Marchetti

IDI IRCCS: Istituto Dermopatico dell'Immacolata Istituto di Ricovero e Cura a Carattere Scientifico Federica De Galitiis

IDI IRCCS: Istituto Dermopatico dell'Immacolata Istituto di Ricovero e Cura a Carattere Scientifico

Cristina M. Failla ( $\sim$ c.failla@idi.it )

IDI IRCCS: Istituto Dermopatico dell'Immacolata Istituto di Ricovero e Cura a Carattere Scientifico https://orcid.org/0000-0001-5991-8914

\section{Cristina Fortes}

IDI IRCCS: Istituto Dermopatico dell'Immacolata Istituto di Ricovero e Cura a Carattere Scientifico

\section{Research Article}

Keywords: vitiligo, leukoderma, melanoma, immune checkpoint inhibitors

Posted Date: August 23rd, 2021 
DOl: https://doi.org/10.21203/rs.3.rs-827134/v1

License: (c) (1) This work is licensed under a Creative Commons Attribution 4.0 International License. Read Full License 


\section{Abstract}

Purpose. Although development of immune checkpoint inhibitors has revolutionized the treatment of metastatic melanoma, more than a half of treated patients experience disease progression during therapy. Cases of spontaneous vitiligo-like leukoderma have been described in melanoma patients and have been associated with a favorable outcome. This vitiligo-like leukoderma can also appear in melanoma patients undergoing immune therapies such as immune checkpoint inhibitors. However, no consensus exists about the relationship between vitiligo-like leukoderma onset and improved overall survival. Our study investigates the possible association between the onset of vitiligo-like leukoderma during immune checkpoint inhibitor treatment and a better prognosis.

Methods. A non-concurrent cohort study was conducted by identifying retrospectively 280 patients who had inoperable or metastatic melanoma and had undergone immune therapy with checkpoint inhibitors in any line of treatment. Toxicities developed during therapy were evaluated.

Results. Among the 280 study participants, 50\% developed at least one type of toxicity, and vitiligo-like leukoderma was observed in 43 patients (15.4\%). In the multivariate Cox model, a protective effect for mortality was observed for patients with vitiligo-like leukoderma development $(\mathrm{HR}=0.23 ; 95 \% \mathrm{Cl}=0.11$ $0.44, p<0.0001)$. In a sub-group analysis comprising only cutaneous melanoma in first line of treatment $(\mathrm{N}=153)$, occurrence of vitiligo-like leukoderma was also an independent predictor factor for duration of clinical benefits measured by time to the next treatment (HR:0.17; 95\% Cl:0.06-0.44).

Conclusion. Our findings indicate that onset of vitiligo-like leukoderma during melanoma treatment could be a marker of favorable outcome in patients treated with immune checkpoint inhibitors.

\section{Introduction}

Treatment of metastatic melanoma has undergone a remarkable evolution in the last few years, leading to the development of innovative therapeutic strategies [1]. Target inhibitors of the mitogen-activated protein kinase pathway, both inhibitors of mutated BRAF and of the mitogen-activated protein kinase (MEK) kinase, on one hand, and the immune checkpoint inhibitors (ICI), either anti-cytotoxic T-lymphocyte antigen (CTLA)-4 (Ipilimumab) or anti-programmed cell death protein 1 (PD-1) (Pembrolizumab or Nivolumab), on the other hand, increased the median progression free survival (PFS) and overall survival (OS) with a portion of long-survivors [2-5]. Despite these encouraging results, more than half of the patients experienced disease progression during treatment. Therefore, it is essential to understand the clinical and physio-pathological features of the patients who achieve the best outcome to define the most appropriate therapeutic strategies. The main risk factor for the development of cutaneous melanoma is exposure to solar or artificial ultraviolet rays which leads to the typical mutational signature with a $\mathrm{C}>\mathrm{T}$ nucleotide transition in dipyrimidine sites [6]. Then, other mutations occur during tumor progression, making melanoma the cancer type with the highest tumor mutational burden (TMB) [7]. Tumors with high TMB have a great probability of creating epitopes that can be presented by dendritic cells, leading to an 
efficient activation of immune cells [8]. Despite this, the host immune system rarely succeeds in spontaneous melanoma clearance. However, presence of a high TMB confers a great advantage in the ICI treatment [9], as it provides a range of antigens against which immune cells, once re-activated by ICl, can act.

In melanoma patients, spontaneous occurrence of cutaneous depigmentation or leukoderma has been reported [10]. It is a clinical manifestation remarkably similar to vitiligo, an autoimmune disease characterized by the presence of autoimmune destruction of cutaneous melanocytes [11] Antigens recognized by cytotoxic $\mathrm{T}$ lymphocytes nearby the leukoderma can be expressed both by normal melanocytes and by melanoma cells, thus explaining the coexistence of the neoplasm and the autoimmune response that determines skin depigmentation. Antigens recognized by $T$ cells are generally characteristic of the melanogenesis process, such as gp100, MART-1, the tyrosinase enzyme or the tyrosine-specific transport protein $1 / 2[12,13]$. Recognition of these antigens by $T$ lymphocytes seldom leads to regression of the cancerous lesions [14].

Development of vitiligo-like leukoderma (VLL) has been described as a side effect of immunomodulating treatments in cutaneous melanoma, starting with vaccine and interleukin (IL)-2 therapies up to modern treatments with ICI [15]. Incidence of VLL in patients treated with anti-CTLA-4 or anti-PD-1 was reported in a very variable range, from $3.4 \%$ and $28.0 \%$, with an onset occurring on average at 30 weeks from inception of treatment $[16,17]$. Indeed, ICI therapy creates an imbalance in immune tolerance that may lead to uncontrolled immune reactions that manifest clinically as autoimmune events [18]. Recent systematic reviews focused on the evaluation of the possible link between immune-related adverse events (irAE) and clinical benefit in patients treated with $\mathrm{ICl}$, analyzing retrospective studies and highlighting the association between autoimmune cutaneous toxicity and favorable outcome [19-21]. Regarding melanoma, a limited number of studies reported an association between development of irAE and better prognosis. This association was further confirmed only for autoimmune cutaneous effects such as VLL $[20,21]$. However, the number of patients included in these studies was small, so that no conclusive evidence could be provided. The aim of our study was to investigate whether an association between the onset of VLL during immune checkpoint inhibitor treatment and a better prognosis exists.

\section{Materials And Methods}

Patients. All patients at IDI-IRCCS, aged $\geq 18$ years, who had inoperable or metastatic melanoma, according to the American Joint Committee on Cancer melanoma staging and classification [22] and who had undergone ICl therapy (anti-PD-1 or anti-CTLA-4) in any line of treatment in the last 10 years, from September 2010 to December 2019, were identified and included in the study. The protocol was approved by the IDI-IRCCS Institutional Ethical Committee (n. 510/3, 2018). Demographic and clinical information was retrieved from medical records. Histological type, tumor thickness, ulceration, and regression were recorded, according to published guidelines [23-26]. The variables considered were: age at diagnosis of primary melanoma, gender, site of the primary melanoma, Breslow thickness, presence of ulceration, lymph node status, presence of regression, presence of satellitosis, age at start of ICI therapy, melanoma 
staging at the start of treatment, number of sites affected by secondary lesions, serum lactate dehydrogenase (LDH), BRAF mutation, specific ICI drug used, line of treatment, use of adjuvant treatment (i.e., interferon-a, ICI), type of toxicity developed during $\mathrm{ICl}$ therapy. Toxicities were evaluated through clinical examination, laboratory, and radiological assessments, using common toxicity grade criteria [27]. Patients affected by vitiligo before initiation of ICI therapy were excluded from the study.

Evaluation. The primary objective was to evaluate the association between VLL onset and the risk of death. Secondary objectives were to evaluate the association between VLL development and time to next treatment (TTNT) and the best overall response in ICl as first-line treatment. TTNT was used as an endpoint since it reflects not only the duration of treatment efficacy, but it also incorporates the patient tolerance to the therapy. Tumor response was assessed using Response Evaluation Criteria in Solid Tumors (RECIST) version 1.1 [28]. The Lazio Region Internet Portal of Health was accessed to obtain official, standardized information on the vital status of all patients. For the overall survival analysis, we calculated the duration of follow-up for each subject as the number of days from the start of the first ICI treatment to the date of death or to 31 December 2019, whichever came first. Time-to-next treatment was calculated as the number of days from the start of the first $\mathrm{ICl}$ treatment to the date of the decision to change treatment because of progression, exhaustion of the clinical benefit, and/or toxicity. For the best overall response, each patient was assigned to one of the following categories: complete response (CR), partial response (PR), stable disease (SD), progressive disease (PD).

Statistical analysis. To compare demographic, histological, clinical characteristics and overall objective response between patients who have developed VLL to those who have not developed this toxicity, the Fisher's exact test was used for the categorical variables, while the Mann-Whitney $U$ test was used for the continuous variables.

For the survival analysis, the Kaplan-Meier method and the log-rank test was used to compare the survival curves between different groups. Time zero corresponds to the start of the first ICl treatment. The Cox proportional-hazard model was used to investigate the association between VLL development and the risk of mortality, and it was also used to examine prognostic factors for TTNT.

For statistical analysis, the STATA software, release 15 (Stata Corp LLC, College Station, TX) was used.

\section{Results}

Two hundred and eighty patients were included in the study. The mean age of the patients at primary melanoma diagnosis was 62.9 years (standard deviation, $S D=14.9$ ), with a median age of 65 years (interquartile range, IQR $=54-74)$. Most patients were males $(60.7 \%$ versus $39.3 \%)$. Median follow-up time from melanoma diagnosis was 3.4 years $(\mathrm{IQR}=1.8-6.2)$ for a total of 1286 person-years of followup. There were 144 deaths; five- and ten-year overall survival were $56.2 \%$ and $30.8 \%$, respectively (Table 1). 
Table 1

Characteristics of primary melanoma

\begin{tabular}{|c|c|c|}
\hline Characteristics: & N. & $\%$ \\
\hline \multicolumn{3}{|l|}{ Age, y } \\
\hline mean (SD) & \multicolumn{2}{|c|}{$62.9(14.9)$} \\
\hline median (IQR) & \multicolumn{2}{|c|}{$65(54-74)$} \\
\hline \multicolumn{3}{|l|}{ Sex } \\
\hline male & 170 & 60.7 \\
\hline female & 110 & 39.3 \\
\hline \multicolumn{3}{|l|}{ Localization of primary } \\
\hline skin & 218 & 77.9 \\
\hline nodular & 96 & 44.0 \\
\hline superficial spreading & 94 & 43.1 \\
\hline other & 28 & 12.8 \\
\hline mucosal & 26 & 9.3 \\
\hline uveal & 9 & 3.2 \\
\hline occult & 12 & 4.3 \\
\hline unknown & 15 & 5.4 \\
\hline \multicolumn{3}{|l|}{ Anatomic site } \\
\hline head/neck & 52 & 18.6 \\
\hline upper limbs & 42 & 15.0 \\
\hline trunk & 100 & 35.7 \\
\hline lower limbs & 59 & 21.1 \\
\hline unknown & 27 & 9.6 \\
\hline \multicolumn{3}{|c|}{ Breslow thickness, $\mathrm{mm}^{\mathrm{a}}$} \\
\hline mean (SD) & \multicolumn{2}{|c|}{$3.9(3.2)$} \\
\hline median (IQR) & \multicolumn{2}{|c|}{$3(2-5)$} \\
\hline \multicolumn{3}{|l|}{ Ulceration $^{\mathrm{a}}$} \\
\hline present & 130 & 59.6 \\
\hline
\end{tabular}




\begin{tabular}{|lll|}
\hline Characteristics: & N. & $\%$ \\
\hline absent & 65 & 29.8 \\
\hline unknown & 23 & 10.6 \\
\hline Regression $^{\text {a }}$ & & \\
\hline yes & 9 & 4.1 \\
\hline no & 209 & 95.9 \\
\hline Presence of satellitosis & \\
\hline yes & & \\
\hline no & 23 & 10.6 \\
\hline Lymphadenectomy & \\
\hline performed & 195 & 89.4 \\
\hline positive & & \\
\hline negative & 102 & 46.8 \\
\hline not performed & 61 & 59.8 \\
\hline Abbreviations: SD, standard deviation; IQR, Interquartile Range \\
a: only for cutaneous melanoma & 41 & 40.2 \\
\hline
\end{tabular}

Regarding the primary melanoma, $77.9 \%$ was of skin onset (of which $44.0 \%$ of nodular type, $43.1 \%$ of superficial diffusion, $12.8 \%$ of other histological type); $9.3 \%$ was mucosal; $3.2 \%$ was uveal and $4.3 \%$ was of unknown origin (occult melanoma). For $5.4 \%$ no data on primary melanoma were available. The most frequent location of the primary melanoma was the trunk (35.7\%), followed by lower extremities (21.1\%), head and neck (18.6\%), and the upper extremities (15.0\%). Breslow thickness was $3.9 \mathrm{~mm}(\mathrm{SD}=3.2)$, with a median of $3 \mathrm{~mm}$ (IQR $=2-5)$. Ulceration was present in $59.6 \%$ of cases, absent in the $29.8 \%$, unknown for a $10.6 \%$. Regression was present only in $4.1 \%$ of cases and the presence of satellitosis in the $10.6 \%$ of cases. One hundred and two $(102,46.8 \%)$ patients had undergone lymphadenectomy, of which $59.8 \%$ were positive and $40.2 \%$ were negative for lymph node metastasis (Table 1 ).

As shown in Table 2, patients at the beginning of treatment with $\mathrm{ICl}$ had a mean age of 66.1 years (SD = $14.1)$, with a median age of 68 years $(\mathrm{IQR}=58-77)$. Out of the 280 patients, $84(30.0 \%)$ were in stage $M 1$, 51 (18.2\%) in stage M1b, 97 (34.6\%) in stage M1c and 47 (16.8\%) in stage M1d. Most of the patients showed the involvement of two metastatic organ sites (35.5\%), followed by one (34.8\%), and by three or more (29.7\%). Serum LDH value was normal in $45.0 \%$ of patients and high in $26.8 \%$. A BRAF mutation was present in $29.6 \%$ of patients (with V600E mutation in $86.7 \%$ of cases, V600K in $12.0 \%$ of cases, one 
case with K601E mutation), and was not present in $66.4 \%$ of patients; for $3.9 \%$ of patients this data was not available. 
Table 2

Patient characteristics at Immune Checkpoint Inhibitors (ICI) therapy baseline

\begin{tabular}{|c|c|c|}
\hline Characteristics: & N. & $\%$ \\
\hline \multicolumn{3}{|l|}{ Age, y } \\
\hline mean (SD) & \multicolumn{2}{|c|}{$66.1(14.1)$} \\
\hline median (IQR) & \multicolumn{2}{|c|}{$68(58-77)$} \\
\hline \multicolumn{3}{|l|}{ Metastatic stage } \\
\hline M1a & 84 & 30.0 \\
\hline M1b & 51 & 18.2 \\
\hline M1c & 97 & 34.6 \\
\hline M1d & 47 & 16.8 \\
\hline unknown & 1 & 0.4 \\
\hline \multicolumn{3}{|c|}{ Number of organs involved } \\
\hline 1 & 97 & 34.8 \\
\hline 2 & 99 & 35.5 \\
\hline$\geq 3$ & 83 & 29.7 \\
\hline \multicolumn{3}{|l|}{ Serum LDH } \\
\hline normal & 126 & 45.0 \\
\hline high $^{\mathrm{a}}$ & 75 & 26.8 \\
\hline unknown & 79 & 28.2 \\
\hline \multicolumn{3}{|l|}{ BRAF status } \\
\hline mutation & 83 & 29.6 \\
\hline V600E & 72 & 86.7 \\
\hline V600K & 10 & 12.0 \\
\hline K601E & 1 & 1.2 \\
\hline no mutation & 186 & 66.4 \\
\hline unknown & 11 & 3.9 \\
\hline
\end{tabular}


As shown in Table 3, two hundred patients $(71.4 \%)$ received ICl therapy as a first-line treatment, 76 $(27.1 \%)$ as a second-line treatment, and only 4 patients (1.4\%) as third- or fourth -line treatment. Among the patients who received $\mathrm{ICl}$ as a first line therapy, 103 patients $(51.5 \%)$ received Nivolumab, 47 Pembrolizumab (23.5\%), and 50 Ipilimumab (25.0\%). Out of 280 patients, 248 (88.6\%) did not receive any adjuvant therapy before $\mathrm{ICI}$ treatment. Among the 280 patients studied, $140(50.0 \%)$ developed at least one type of toxicity. The following toxicities were observed: gastro-intestinal (19.3\%); endocrinological (15.4\%); VLL (15.4\%); skin toxicity excluding VLL (11.4\%); pulmonary (4.3\%); skeletal muscle (2.5\%); neurological (1.4\%); cardiovascular ( $0.7 \%)$; hematological $(0.7 \%)$; ocular $(0.7 \%)$ and renal toxicity $(0.4 \%)$. It is important to note that, among the 43 patients who developed VLL, 25 patients (58.1\%) did not have any other toxicity. The median time of VLL onset after the start of Ipilimumab, Pembrolizumab and Nivolumab as a first-line treatment was 7 months (IQR = 3-14), 6 months (IQR =4-8), and 10 months (417) respectively. No difference was found between ICI treatment types and onset of VLL $(p=0.713)$. From the start of ICl therapy, the median follow-up time was 9.9 months (ranging from 6 days to 81 months) for a total of 4651 months of follow-up. 
Table 3

Type of therapies and toxicity

\begin{tabular}{|c|c|c|}
\hline & $\mathbf{N}$. & $\%$ \\
\hline \multicolumn{3}{|l|}{$\mathrm{ICl}$ line of treatment } \\
\hline first-line & 200 & 71.4 \\
\hline second-line & 76 & 27.1 \\
\hline third or fourth line & 4 & 1.4 \\
\hline \multicolumn{3}{|l|}{ First-line drug } \\
\hline Ipilimumab & 50 & 25.0 \\
\hline Pembrolizumab & 47 & 23.5 \\
\hline Nivolumab & 103 & 51.5 \\
\hline \multicolumn{3}{|l|}{ Adjuvant therapy } \\
\hline no & 248 & 88.6 \\
\hline yes & 30 & 10.7 \\
\hline unknown & 2 & 0.7 \\
\hline \multicolumn{3}{|l|}{ Toxicity } \\
\hline no & 140 & 50.0 \\
\hline yes & 140 & 50.0 \\
\hline \multicolumn{3}{|l|}{ Type of toxicity } \\
\hline leukoderma & 43 & 15.4 \\
\hline only leukoderma & 25 & 8.9 \\
\hline gastro-intestinal & 54 & 19.3 \\
\hline endocrine & 43 & 15.4 \\
\hline cutaneous (excluding leukoderma) & 32 & 11.4 \\
\hline pulmonary & 12 & 4.3 \\
\hline skeletal muscle & 7 & 2.5 \\
\hline neurological & 4 & 1.4 \\
\hline cardiovascular & 2 & 0.7 \\
\hline hematological & 2 & 0.7 \\
\hline
\end{tabular}




\begin{tabular}{|lcc|}
\hline & N. & $\%$ \\
\hline ocular & 2 & 0.7 \\
\hline renal & 1 & 0.4 \\
\hline Abbreviations. ICl, Immune Checkpoint Inhibitors \\
\hline
\end{tabular}

A significant survival advantage in patients who developed VLL during ICl treatment compared to those who did not develop such a toxicity was observed (log-rank test, $p<0.0001$; Fig. 1, panel A). Five-year overall survival for persons who developed VLL was $75.1 \%$ versus $24.2 \%$ for those who did not develop it. This effect was only observed for VLL and not for other toxicities, regardless of the toxicity grade. A significant poorer survival was found for patients with high LDH compared to those with normal serum LDH levels (log-rank test, $p<0.0001$; Fig. 1, panel B); with metastatic disease M1c and M1d compared to M1a-M1b (log-rank test, $p<0.0001$; Fig. 2, panel A); and in patients who did not undergo previous adjuvant treatment compared to those who did (log-rank test, $p=0.047$; Fig. 2, panel B).

Table 4 shows the results of the multivariate analysis for mortality. After controlling for sex, age, staging, LDH levels, use of adjuvant therapy, first line use of ICls, VLL development was associated with reduced mortality (hazard ratio, HR:0.32; 95\% Cl: $0.22-0.48, p<0.0001$ ). The protective effect was even greater if the analysis was restricted to patients with cutaneous melanoma who underwent treatment with $\mathrm{ICl}$ in first line (HR:0.24; 95\% Cl: 0.12-0.45, p < 0.0001). For patients in which ICl was the first line therapy and VLL was the only toxicity observed, mortality risk was 6 times lower than in patients with no toxicity (HR:0.15; 95\% Cl = 0.05-0.43, $p<0.0001$ ). High serum LDH levels (HR:2.33; 95\% Cl:1.51-3.60, $p<0.0001$ ), staging (M1d versus M1a-1b) (HR:2.25; 95\% Cl: 1.39-3.63, $p=0.001)$ and use of ICls not as a first line treatment (HR:1.47; 95\% Cl:1.02-2.13, $\mathrm{p}=0.040)$, were all independently associated with an increased risk of mortality in the multivariate model, while the use of adjuvant therapy was associated with a protective effect (HR:0.54; 95\%Cl: 0.29-0.99, $p=0.047)$. 
Table 4

Prognostic factors for mortality: uni- and multi-variate Cox model

\begin{tabular}{|c|c|c|c|c|c|c|c|c|}
\hline & \multicolumn{4}{|c|}{$\begin{array}{l}\text { all melanoma } \\
(\mathrm{N}=280)\end{array}$} & \multicolumn{2}{|c|}{$\begin{array}{l}\text { only cutaneous } \\
\text { melanoma } \\
(\mathrm{N}=218)\end{array}$} & \multicolumn{2}{|c|}{$\begin{array}{l}\text { only cutaneous } \\
\text { melanoma and } \\
\text { first-line ICl } \\
(\mathrm{N}=153)\end{array}$} \\
\hline & $\begin{array}{l}\text { HRcrude } \\
(95 \% \mathrm{Cl})\end{array}$ & $P$ & $\begin{array}{l}\text { HRadja } \\
(95 \% \mathrm{Cl})\end{array}$ & $P$ & $\begin{array}{l}\text { HRadja } \\
(95 \% \mathrm{Cl})\end{array}$ & $P$ & $\begin{array}{l}\text { HRadja } \\
(95 \% \mathrm{Cl})\end{array}$ & $P$ \\
\hline \multicolumn{9}{|l|}{ Sex } \\
\hline male & 1 & & 1 & & 1 & & 1 & \\
\hline female & $\begin{array}{l}0.78 \\
(0.56- \\
1.10)\end{array}$ & 0.162 & $\begin{array}{l}0.74 \\
(0.51- \\
1.07)\end{array}$ & 0.110 & $\begin{array}{l}0.75 \\
(0.49- \\
1.16)\end{array}$ & 0.198 & $\begin{array}{l}0.82 \\
(0.45- \\
1.48)\end{array}$ & 0.505 \\
\hline $\begin{array}{l}\text { Age at start } \\
\text { of ICl }\end{array}$ & $\begin{array}{l}1.00 \\
(0.99- \\
1.02)\end{array}$ & 0.658 & $\begin{array}{l}1.01 \\
(1.00- \\
1.03)\end{array}$ & 0.076 & $\begin{array}{l}1.01 \\
(0.99- \\
1.03)\end{array}$ & 0.190 & $\begin{array}{l}1.04 \\
(1.01- \\
1.06)\end{array}$ & 0.002 \\
\hline \multicolumn{9}{|l|}{ Toxicity } \\
\hline none & 1 & & 1 & & 1 & & 1 & \\
\hline $\begin{array}{l}\text { leukoderma } \\
\text { and other }\end{array}$ & $\begin{array}{l}0.26 \\
(0.18- \\
0.38)\end{array}$ & $\begin{array}{l}< \\
0.0001\end{array}$ & $\begin{array}{l}0.32 \\
(0.22- \\
0.48)\end{array}$ & $\begin{array}{l}< \\
0.0001\end{array}$ & $\begin{array}{l}0.28 \\
(0.17- \\
0.45)\end{array}$ & $\begin{array}{l}< \\
0.0001\end{array}$ & $\begin{array}{l}0.24 \\
(0.12- \\
0.45)\end{array}$ & $\begin{array}{l}< \\
0.0001\end{array}$ \\
\hline $\begin{array}{l}\text { only } \\
\text { leukoderma }\end{array}$ & $\begin{array}{l}0.23 \\
(0.12- \\
0.44)\end{array}$ & $\begin{array}{l}<.0001 \\
0.00\end{array}$ & $\begin{array}{l}0.23 \\
(0.11- \\
0.44)\end{array}$ & $\begin{array}{l}<.0001 \\
0.0\end{array}$ & $\begin{array}{l}0.22 \\
(0.11- \\
0.46)\end{array}$ & $\begin{array}{l}<.0001 \\
0.0\end{array}$ & $\begin{array}{l}0.15 \\
(0.05- \\
0.43)\end{array}$ & $<0.0001$ \\
\hline \multicolumn{9}{|l|}{ Serum LDH } \\
\hline normal & 1 & & 1 & & 1 & & & \\
\hline high $^{a}$ & $\begin{array}{l}2.75 \\
(1.84- \\
4.10)\end{array}$ & $\begin{array}{l}< \\
0.0001\end{array}$ & $\begin{array}{l}2.33 \\
(1.51- \\
3.60)\end{array}$ & $\begin{array}{l}< \\
0.0001\end{array}$ & $\begin{array}{l}2.76 \\
(1.66- \\
4.58)\end{array}$ & $\begin{array}{l}< \\
0.0001\end{array}$ & $\begin{array}{l}2.75 \\
(1.46- \\
5.16)\end{array}$ & $\begin{array}{l}< \\
0.0001\end{array}$ \\
\hline \multicolumn{9}{|l|}{$\begin{array}{l}\text { Metastatic } \\
\text { stage }\end{array}$} \\
\hline $1 a-1 b$ & 1 & & 1 & & 1 & & 1 & \\
\hline $1 c$ & $\begin{array}{l}2.21 \\
(1.51- \\
3.23)\end{array}$ & $\begin{array}{l}< \\
0.0001\end{array}$ & $\begin{array}{l}1.44 \\
(0.96- \\
2.16)\end{array}$ & 0.075 & $\begin{array}{l}1.52 \\
(0.95- \\
2.43)\end{array}$ & 0.084 & $\begin{array}{l}1.92 \\
(1.01- \\
3.64)\end{array}$ & 0.047 \\
\hline $1 d$ & $\begin{array}{l}3.22 \\
(2.08- \\
4.99)\end{array}$ & $\begin{array}{l}< \\
0.0001\end{array}$ & $\begin{array}{l}2.25 \\
(1.39- \\
3.63)\end{array}$ & 0.001 & $\begin{array}{l}1.76 \\
(1.00- \\
3.10)\end{array}$ & 0.051 & $\begin{array}{l}2.80 \\
(1.36- \\
5.78)\end{array}$ & 0.005 \\
\hline
\end{tabular}




\begin{tabular}{|c|c|c|c|c|c|c|c|c|}
\hline yes vs. no & $\begin{array}{l}0.55 \\
(0.31- \\
1.00)\end{array}$ & 0.051 & $\begin{array}{l}0.54 \\
(0.29- \\
0.99)\end{array}$ & 0.047 & $\begin{array}{l}0.47 \\
(0.25- \\
0.91)\end{array}$ & 0.024 & $\begin{array}{l}1.22 \\
(0.57- \\
2.61)\end{array}$ & 0.605 \\
\hline \multicolumn{9}{|l|}{$\begin{array}{l}\text { ICI line of } \\
\text { treatment }\end{array}$} \\
\hline first-line & 1 & & 1 & & 1 & & - & \\
\hline $\begin{array}{l}\text { second-line } \\
\text { and beyond }\end{array}$ & $\begin{array}{l}1.94 \\
(1.39- \\
2.72)\end{array}$ & $\dot{0} 0001$ & $\begin{array}{l}1.47 \\
(1.02- \\
2.13)\end{array}$ & 0.040 & $\begin{array}{l}1.53 \\
(1.00- \\
2.33)\end{array}$ & 0.047 & - & \\
\hline \multicolumn{9}{|c|}{$\begin{array}{l}\text { Abbreviations: HR, Hazard Ratio; adj, adjusted; Cl, Confidence Intervals; LDH, Lactate Dehydrogenase; } \\
\text { ICl, Immune Checkpoint Inhibitors }\end{array}$} \\
\hline \multicolumn{9}{|c|}{ a: HR adjusted for all items in table } \\
\hline \multicolumn{9}{|c|}{ b: > upper limit of normal (ULN) } \\
\hline
\end{tabular}

A subgroup analysis was conducted only for patients with ICls as a first line therapy $(N=153)$. The median TTNT was 4.9 months. The occurrence of VLL was also an independent predictor factor for duration of clinical benefits measured by TTNT (only VLL), HR:0.17; 95\%Cl:0.06-0.44, $p=<0.0001$; VLL and other toxicities HR:0.35; $95 \% \mathrm{Cl}: 0.21-0.59, \mathrm{p}=<0.0001$. Both high LDH serum levels and advance staging were associated with shorter TTNT (Table 5). 
Table 5

Prognostic factors for time to the next treatment (TTNT): uni- and multi-variate Cox model

\begin{tabular}{|c|c|c|c|c|}
\hline & \multicolumn{4}{|c|}{$\begin{array}{l}\text { only cutaneous melanoma and first-line ICI } \\
(N=153)\end{array}$} \\
\hline & HRcrude $(95 \% \mathrm{Cl})$ & $P$ & $\operatorname{HRadj}^{\mathrm{a}}(95 \% \mathrm{Cl})$ & $P$ \\
\hline \multicolumn{5}{|l|}{ Sex } \\
\hline male & 1 & & 1 & \\
\hline female & $0.96(0.61-1.49)$ & 0.846 & $1.02(0.63-1.66)$ & 0.934 \\
\hline Age at start of ICl & $1.00(0.99-1.02)$ & 0.564 & $1.01(0.99-1.02)$ & 0.513 \\
\hline \multicolumn{5}{|l|}{ Toxicity } \\
\hline none & 1 & & 1 & \\
\hline leukoderma and other & $0.32(0.20-0.51)$ & $<0.0001$ & $0.35(0.21-0.59)$ & $<0.0001$ \\
\hline only leukoderma & $0.19(0.07-0.48)$ & $<0.0001$ & $0.17(0.06-0.44)$ & $<0.0001$ \\
\hline \multicolumn{5}{|l|}{ Serum LDH } \\
\hline normal & 1 & & 1 & \\
\hline high $^{b}$ & $1.95(1.19-3.20)$ & 0.008 & $2.06(1.19-3.59)$ & 0.010 \\
\hline \multicolumn{5}{|l|}{ Metastatic stage } \\
\hline M1a-1b & 1 & & 1 & \\
\hline M1c & $1.93(1.15-3.24)$ & 0.013 & $1.53(0.88-2.65)$ & 0.130 \\
\hline M1d & $2.84(1.66-4.88)$ & $<0.0001$ & $2.08(1.14-3.79)$ & 0.017 \\
\hline \multicolumn{5}{|l|}{ Adjuvant therapy } \\
\hline yes vs. no & $1.20(0.65-2.22)$ & 0.553 & $1.35(0.70-2.60)$ & 0.364 \\
\hline $\begin{array}{l}\text { Abbreviations: HR, Haze } \\
\text { a: HR adjusted for all it } \\
\text { b: > upper limit of norm }\end{array}$ & $\begin{array}{l}\text { atio; adj, adjusted; } \\
\text { n table } \\
\text { N) }\end{array}$ & nfidence It & vals; LDH, Lactate & drogenase \\
\hline
\end{tabular}

All other factors considered in the study, such as Breslow thickness, ulceration, site of primary melanoma, presence of regression, presence of satellitosis, lymphadenectomy, BRAF status, and types of ICI used in first line (Ipilimumab, Pembrolizumab or Nivolumab), were not associated with mortality. 
Demographic and clinical characteristics of patients who developed VLL and of the ones who do not developed it were examined (Table 6). No difference between groups were found for age, types of melanoma, anatomic site of the primary melanoma, BRAF status, use of adjuvant therapy, and type of ICI. However, statistically significant differences were observed for sex $(p=0.043)$, and LDH levels $(p=0.021)$. Most patients who developed VLL were females (53.5\% versus $36.7 \%$ ) and had normal serum LDH levels $(80.0 \%$ versus $59.0 \%)$. For patients with $\mathrm{ICl}$ as first-line treatment, the best objective response was also analyzed. The rates of clinical complete responses (CR) (35.3\% versus 9.1\%) and partial responses (PR) (38.2\% versus $28.0 \%$ ) were higher in patients who developed VLL than in patients who did not have such a toxicity, while progressive disease (PD) was significantly lower in patients with VLL (5.9\% versus $40.9 \%$, $p<0.0001)$. 
Table 6

Demographic and clinical characteristics of the patients and onset of leukoderma

\begin{tabular}{|c|c|c|c|c|c|}
\hline \multirow[b]{2}{*}{ Characteristics: } & \multicolumn{2}{|c|}{$\begin{array}{l}\text { leukoderma } \\
(\mathrm{N}=43)\end{array}$} & \multicolumn{2}{|c|}{$\begin{array}{l}\text { no leukoderma } \\
(\mathrm{N}=237)\end{array}$} & \multirow[b]{2}{*}{$P^{a}$} \\
\hline & N.* & $\%$ & N.* & $\%$ & \\
\hline \multicolumn{6}{|l|}{ Age at start of ICl } \\
\hline mean $(S D)$ & \multicolumn{2}{|c|}{$66.2(12.7)$} & \multicolumn{2}{|c|}{$66.1(14.4)$} & $0.831^{b}$ \\
\hline \multicolumn{6}{|l|}{ Sex } \\
\hline male & 20 & 46.5 & 150 & 63.3 & \\
\hline female & 23 & 53.5 & 87 & 36.7 & 0.043 \\
\hline \multicolumn{6}{|c|}{ Localization of primary } \\
\hline Cutaneous & 35 & 83.3 & 183 & 82.1 & \\
\hline Mucosal & 4 & 9.5 & 22 & 9.9 & \\
\hline Uveal & 0 & $\ldots$ & 9 & 4.0 & \\
\hline Occult & 3 & 7.1 & 9 & 4.0 & 0.537 \\
\hline \multicolumn{6}{|l|}{ Anatomic site } \\
\hline head/neck & 5 & 13.2 & 47 & 21.9 & \\
\hline upper limbs & 3 & 7.9 & 39 & 18.1 & \\
\hline trunk & 16 & 42.1 & 84 & 39.1 & \\
\hline lower limbs & 14 & 36.8 & 45 & 20.9 & 0.089 \\
\hline \multicolumn{6}{|l|}{ Metastatic stage } \\
\hline M1a-1b & 28 & 65.1 & 107 & 45.3 & \\
\hline M1c & 10 & 23.3 & 87 & 36.9 & \\
\hline M1d & 5 & 11.6 & 42 & 17.8 & 0.071 \\
\hline \multicolumn{6}{|l|}{ Serum LDH } \\
\hline normal & 28 & 80.0 & 98 & 59.0 & \\
\hline high $^{a}$ & 7 & 20.0 & 68 & 41.0 & 0.021 \\
\hline \multicolumn{6}{|l|}{ BRAF status } \\
\hline mutation & 29 & 69.0 & 157 & 69.2 & \\
\hline
\end{tabular}




\begin{tabular}{|c|c|c|c|c|c|}
\hline \multirow{2}{*}{ no mutation } & \multicolumn{2}{|c|}{$\begin{array}{l}\text { leukoderma } \\
(\mathrm{N}=43)\end{array}$} & \multicolumn{2}{|c|}{$\begin{array}{l}\text { no leukoderma } \\
(\mathrm{N}=237)\end{array}$} & \multirow[b]{2}{*}{1.00} \\
\hline & 13 & 31.0 & 70 & 30.8 & \\
\hline \multicolumn{6}{|l|}{ Adjuvant therapy } \\
\hline no & 35 & 81.4 & 213 & 90.6 & \\
\hline yes & 8 & 18.6 & 22 & 9.4 & 0.104 \\
\hline \multicolumn{6}{|l|}{ First-line drug } \\
\hline Ipilimumab & 7 & 20.6 & 43 & 25.9 & \\
\hline Pembrolizumab & 9 & 26.5 & 38 & 22.9 & \\
\hline Nivolumab & 18 & 52.9 & 85 & 51.2 & 0.801 \\
\hline \multicolumn{6}{|l|}{ Best objective response $^{d}$} \\
\hline Complete Response & 12 & 35.3 & 15 & 9.1 & \\
\hline Partial Response & 13 & 38.2 & 46 & 28.0 & \\
\hline Stable Disease & 7 & 20.6 & 36 & 22.0 & \\
\hline Progressive Disease & 2 & 5.9 & 67 & 40.9 & $<0.0001$ \\
\hline $\begin{array}{l}\text { Abbreviations. SD, Standard Dev } \\
\text { Inhibitors } \\
\text { a: Fisher's exact test } \\
\text { b: Mann-Whitney U test } \\
\text { c: > upper limit of normal (ULN) } \\
\text { d: to first-line therapy }\end{array}$ & LDH & tate De & gena & Immun & kpoint \\
\hline
\end{tabular}

\section{Discussion}

In recent years, treatment of metastatic melanoma has undergone a sudden and encouraging evolution. Search for effective therapies to improve the prognosis of patients with inoperable melanoma has led to the development of innovative drugs, including CTLA-4 and PD-1/PD-ligand-1 (PD-L1) inhibitors, used alone or in combination. Despite these reassuring results, many patients have primary resistance to such treatments, obtaining limited benefit from these therapeutic strategies. Understanding clinical aspects related to treatment response would allow the selection of patients who would truly benefit from these therapies. The possible link between the onset of adverse events and the response to $\mathrm{ICI}$ treatment is one of the subjects of greatest interest in oncology, due to the hypothesis that greater toxicity would also indicate greater anti-neoplastic effect. 
One of the side-effect signs that appears to be related to a better response to ICl treatment in cutaneous melanoma is VLL development. Indeed, some studies suggest the existence of a link between development of VLL and a better outcome in patients treated with $\mathrm{ICI}[20,21]$. However, these studies were conducted in a small number of patients and no multivariate analysis was performed to control for other known prognostic factors of progression (e.g. LDH, metastatic stage). Thus, no firm conclusions could be drawn $[20,21]$.

To further verify the existence of this association, we performed a retrospective analysis on 280 metastatic melanoma patients who had undergone at least one $\mathrm{ICI}$ treatment. Our findings confirm, after controlling for confounding factors, a significant association between VLL development and a better prognosis in terms of overall survival and TTNT that agrees with other studies published elsewhere [20, 21]. The effect was even stronger for patients in which no other toxicity, other than VLL. The latter finding could be related to a probable greater use of high-dose corticosteroids in patients who developed other autoimmune toxicities with consequent partial anti-inflammatory and immunosuppressive effects, as well as to the higher probability of discontinuing treatment in the presence of other toxicities. Indeed, VLL development itself is devoid of clinical significance, except for its psychological impact that could alter patient quality of life.

In our study 43 out of 280 patients (15.4\%), developed VLL which is lower in comparison with other studies $[20,21]$. In our study the median time of VLL onset after the start of Ipilimumab, Pembrolizumab and Nivolumab as a first-line treatment was 7 months (IQR = 3-14), 6 months (IQR = 4-8), and 10 months (4-17) respectively which is in agreement with a previous study [29].

Our data show that complete objective response was reached by $35.3 \%$ of the patients who developed VLL while in the study by Nakamura (2017), conducted on 35 unresectable stage III or IV melanoma patients treated with nivolumab, 9 patients (25.7\%) developed VLL and objective response was $22.2 \%$ among patients with VLL. Hua (2016) conducted a study on 67 unresectable stage III or IV melanoma patients treated with Pembrolizumab. During the treatment with Pembrolizumab VLL appeared in 17 (25\%) patients and complete objective response was $18 \%$ among patients who developed VLL. The median overall survival was 18.2 months in the group of patients that developed VLL compared to 8.5 months in the group of non VLL. In our study median overall survival in the group of patients that developed VLL was 34.0 months vs 8 months in the group of non VLL.

LDH within normal limits, M1a and/or M1b disease, a previous adjuvant treatment, remained independent factors associated with a better prognosis in terms of survival, in line with literature data [30]. The analysis of patients' clinical characteristics showed an association with gender, disease burden, metastatic sites, and the likelihood of VLL onset. Patients of female sex, with metastatic disease involving only skin, lymph nodes or lungs, and/or with serum LDH within the normal limits, were more likely to develop VLL. This evidence could be explained by the fact that a greater aggressiveness of tumor disease, and the presence of visceral and/or encephalic metastases, could underlie a lower ability to develop an effective immune reaction. It is known that some metastatic niches, such as those in the liver 
and brain, have a greater ability to evade the immune response due to a more immunosuppressive environment [31,32]. Regarding gender, female sex is known to be associated with the ability to develop a more efficient immune response [33]. A higher probability of longer progression-free survival in female patients was also reported in a recent multicenter study that analyzed clinical and biological features of melanoma patients who developed VLL after ICl treatment [34]. Differently from our present study, the recent paper from Guida et al. (2021) did not match the VLL patient subset with one without VLL and could not obtain conclusive data about significance of VLL development in the entire population.

It is important to note that this is the largest observational study conducted so far that shows an independent association between VLL development and better prognosis measured by objective overall survival, overall response and TTNT.

In conclusion, our study confirms, on a large patient cohort of advanced cutaneous melanoma, that VLL development as an $\mathrm{ICl}$ toxicity is an independent marker of a more favorable prognosis. Therefore, VLL onset should be considered as a sign of positive response to $\mathrm{ICl}$ treatment. Future studies of the biological mechanisms and of the actual causal chain leading to VLL development, would clarify the different degrees of response to $\mathrm{ICl}$ treatment, possibly leading to an optimization of the therapeutic strategies.

\section{Abbreviations}

MEK: mitogen-activated protein kinase; ICl: immune checkpoint inhibitors; CTLA-4: cytotoxic T-lymphocyte antigen-4; PD-1: programmed cell death protein 1; PFS: progression free survival; OS: overall survival; TMB: tumor mutational burden; VLL: vitiligo-like leukoderma; IL: interleukin; irAE: immune-related adverse events; LDH: lactate dehydrogenase; TTNT: time to next treatment; RECIST: Response Evaluation Criteria in Solid Tumors; CR: complete response; PR: partial response; SD: stable disease; PD: progressive disease; SD: standard deviation; IQR: interquartile range; HR: hazard ratio; PD-L1: PD-ligand-1.

\section{Declarations}

\section{Funding}

This project was funded, in part, with grants from the "Progetto Ricerca Corrente", RC20_4.1, of the Italian Ministry of Health. MLC is the recipient of a Young Investigator Fellowship from Fondazione Umberto Veronesi.

\section{Conflicts of interest}

No competing interests

\section{Availability of data and material}

All relevant data are within the papers 


\section{Authors' contribution}

Conceptualization, SD, FDG, CMF, CF; data collection and management, SV, FRDP, SM, MLC, RM, FMM; formal analysis, SV, FRDP, SM, MLC; methodology, SV, FRDP, SM, MLC, CF; validation, SM, CF; writing original draft, SV, FRDP, MLC, CMF, CF; writing review and editing, DA, RM, FMM, SD, PM, FDG, CMF, CF; funding acquisition, CMF, CF, DA, FDG, PM.

\section{Ethics approval and consent to participate.}

The protocol was approved by the IDIIIRCCS Institutional Ethical Committee (n. 510/3, 2018).

\section{Consent for publication}

Not applicable

\section{Acknowledgements}

This project was funded, in part, with grants from the "Progetto Ricerca Corrente", RC20_4.1, of the Italian Ministry of Health. MLC is the recipient of a Young Investigator Fellowship from Fondazione Umberto Veronesi. Authors would like to acknowledge the efforts and care of the Oncology Department nurse team, and the contribution and compliance of the patients.

\section{References}

1. Maverakis E, Cornelius LA, Bowen GM, Phan T, Patel FB, Fitzmaurice S, et al. Metastatic melanoma A review of current and future treatment options. Acta Derm. Venereol. 2015. p. 516-24.

2. Robert C, Grob JJ, Stroyakovskiy D, Karaszewska B, Hauschild A, Levchenko E, et al. Five-Year Outcomes with Dabrafenib plus Trametinib in Metastatic Melanoma. N Engl J Med. 2019;381:62636.

3. Dreno B, Ascierto PA, McArthur GA, Atkinson V, Liszkay G, Di Giacomo AM, et al. Efficacy and safety of cobimetinib (C) combined with vemurafenib (V) in patients (pts) with BRAF V600 mutationpositive metastatic melanoma: analysis from the 4-year extended follow-up of the phase 3 coBRIM study. J Clin Oncol. 2018;36:9522-9522.

4. Robert C, Ribas A, Schachter J, Arance A, Grob JJ, Mortier L, et al. Pembrolizumab versus ipilimumab in advanced melanoma (KEYNOTE-006): post-hoc 5-year results from an open-label, multicentre, randomised, controlled, phase 3 study. Lancet Oncol. 2019;20:1239-51.

5. Larkin J, Chiarion-Sileni V, Gonzalez R, Grob JJ, Cowey CL, Lao CD, et al. Combined Nivolumab and Ipilimumab or Monotherapy in Untreated Melanoma. N Engl J Med. 2015;373:23-34.

6. León-Letelier RA, Bonifaz LC, Fuentes-Pananá EM. OMIC signatures to understand cancer immunosurveillance and immunoediting: Melanoma and immune cells interplay in immunotherapy. J. Leukoc. Biol. 2019. p. 915-33. 
7. Büttner R, Longshore JW, López-Ríos F, Merkelbach-Bruse S, Normanno N, Rouleau E, et al. Implementing TMB measurement in clinical practice: Considerations on assay requirements. ESMO Open. 2019.

8. Chabanon RM, Pedrero M, Lefebvre C, Marabelle A, Soria JC, Postel-Vinay S. Mutational landscape and sensitivity to immune checkpoint blockers. Clin. Cancer Res. 2016. p. 4309-21.

9. Goodman AM, Kato S, Bazhenova L, Patel SP, Frampton GM, Miller V, et al. Tumor mutational burden as an independent predictor of response to immunotherapy in diverse cancers. Mol Cancer Ther. 2017;16:2598-608.

10. Failla CM, Carbone ML, Fortes C, Pagnanelli G, D'atri S. Melanoma and vitiligo: In good company. Int J Mol Sci. 2019;20.

11. Mason CP, Gawkrodger DJ. Vitiligo presentation in adults. Clin Exp Dermatol. 2005;30:344-5.

12. Rodrigues M, Ezzedine K, Hamzavi I, Pandya AG, Harris JE. New discoveries in the pathogenesis and classification of vitiligo. J Am Acad Dermatol. 2017;77:1-13.

13. Teulings HE, Willemsen KJ, Glykofridis I, Krebbers G, Komen L, Kroon MW, et al. The antibody response against MART-1 differs in patients with melanoma-associated leucoderma and vitiligo. Pigment Cell Melanoma Res. 2014;27:1086-96.

14. Parmiani G. Melanoma antigens and their recognition by T cells. Keio J. Med. 2001. p. 86-90.

15. Teulings HE, Limpens J, Jansen SN, Zwinderman AH, Reitsma JB, Spuls PI, et al. Vitiligo-like depigmentation in patients with stage III-IV melanoma receiving immunotherapy and its association with survival: A systematic review and meta-analysis. J Clin Oncol. 2015;33:773-81.

16. Belum VR, Benhuri B, Postow MA, Hellmann MD, Lesokhin AM, Segal NH, et al. Characterisation and management of dermatologic adverse events to agents targeting the PD-1 receptor. Eur $\mathrm{J}$ Cancer. 2016;60:12-25.

17. Hwang SJE, Park JJW, Wakade D, Chou S, Byth K, Fernandez-Penas P. Cutaneous adverse events of anti-programmed death 1 antibodies combined with anti-cytotoxic T-lymphocyte-Associated protein 4 therapy use in patients with metastatic melanoma. Melanoma Res. 2019;29:172-7.

18. Simeone E, Grimaldi AM, Festino L, Trojaniello C, Vitale MG, Vanella V, et al. Immunotherapy in metastatic melanoma: a novel scenario of new toxicities and their management. Melanoma Manag. 2019;6:МMT30.

19. Cortellini A, Buti S, Agostinelli V, Bersanelli M. A systematic review on the emerging association between the occurrence of immune-related adverse events and clinical outcomes with checkpoint inhibitors in advanced cancer patients. Semin. Oncol. 2019. p. 362-71.

20. Hua C, Boussemart L, Mateus C, Routier E, Boutros C, Cazenave H, et al. Association of vitiligo with tumor response in patients with metastatic melanoma treated with pembrolizumab. JAMA Dermatology. 2016;152:45-51.

21. Nakamura Y, Tanaka R, Asami Y, Teramoto Y, Imamura T, Sato S, et al. Correlation between vitiligo occurrence and clinical benefit in advanced melanoma patients treated with nivolumab: A multiinstitutional retrospective study. J Dermatol. 2017;44:117-22. 
22. Gershenwald JE, Scolyer RA. Melanoma Staging: American Joint Committee on Cancer (AJCC) 8th Edition and Beyond. Ann. Surg. Oncol. 2018. p. 2105-10.

23. Clark WH, From L, Bernardino EA, Mihm MC. The Histogenesis and Biologic Behavior of Primary Human Malignant Melanomas of the Skin. Cancer Res. 1969;29.

24. Clark WH, Elder DE, Guerry D, Braitman LE, Trock BJ, Schultz D, et al. Model predicting survival in stage I melanoma based on tumor progression. J Natl Cancer Inst. 1989;81:1893-904.

25. Breslow A. Thickness, cross-sectional areas and depth of invasion in the prognosis of cutaneous melanoma. Ann Surg. 1970;172:902-8.

26. Busam KJ, Barnhill RL. Melanocytes. Pathol Melanocytic Nevi Malig Melanoma. Springer New York; 2004. p. 1-10.

27. Cancer Institute N. Common Terminology Criteria for Adverse Events (CTCAE) Common Terminology Criteria for Adverse Events (CTCAE) v5.0. 2017.

28. Eisenhauer EA, Therasse P, Bogaerts J, Schwartz LH, Sargent D, Ford R, et al. New response evaluation criteria in solid tumours: Revised RECIST guideline (version 1.1). Eur J Cancer. 2009;45:228-47.

29. Babai S, Voisin AL, Bertin C, Gouverneur A, Le-Louet H. Occurrences and Outcomes of Immune Checkpoint Inhibitors-Induced Vitiligo in Cancer Patients: A Retrospective Cohort Study. Drug Saf. 2020;43:111-7.

30. Keung EZ, Balch CM, Thompson JF, Kirkwood JM, Scolyer RA, Sondak VK, et al. Melanoma Prognosis and Staging. Cutan Melanoma. 2020. p. 271-97.

31. Tumeh PC, Hellmann MD, Hamid O, Tsai KK, Loo KL, Gubens MA, et al. Liver metastasis and treatment outcome with anti-PD-1 monoclonal antibody in patients with melanoma and NSCLC. Cancer Immunol Res. 2017;5:417-24.

32. Di Giacomo AM, Valente M, Cerase A, Lofiego MF, Piazzini F, Calabrò L, et al. Immunotherapy of brain metastases: Breaking a "dogma." J. Exp. Clin. Cancer Res. 2019.

33. Klein SL, Flanagan KL. Sex differences in immune responses. Nat. Rev. Immunol. 2016. p. 626-38.

34. Guida M, Strippoli S, Maule M, Quaglino P, Ramondetta A, Chiaron Sileni V, et al. Immune checkpoint inhibitor associated vitiligo and its impact on survival in patients with metastatic melanoma: an Italian Melanoma Intergroup study. ESMO Open. 2021;6:100064.

\section{Figures}


A

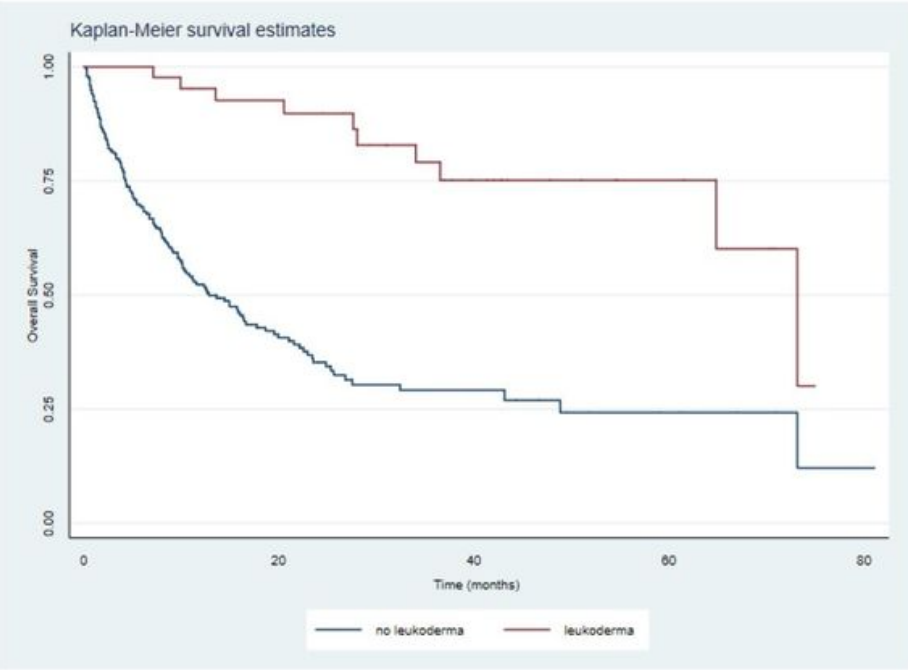

B

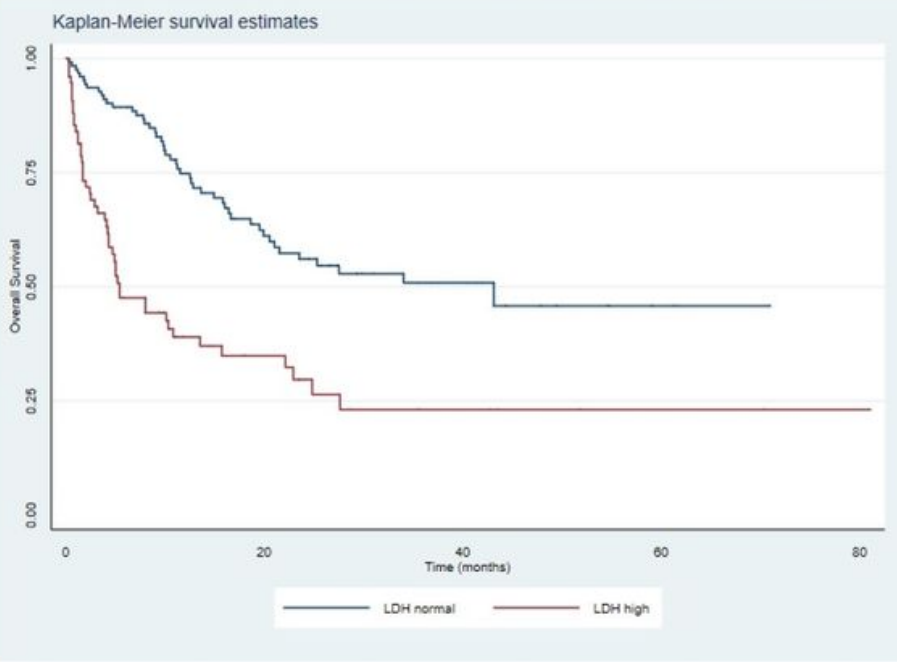

Figure 1

Overall survival analysis. Kaplan-Meier survival curves for analysis in patients who had developed or not VLL during ICl treatment (A), with normal or elevated LDH serum levels (B).

A

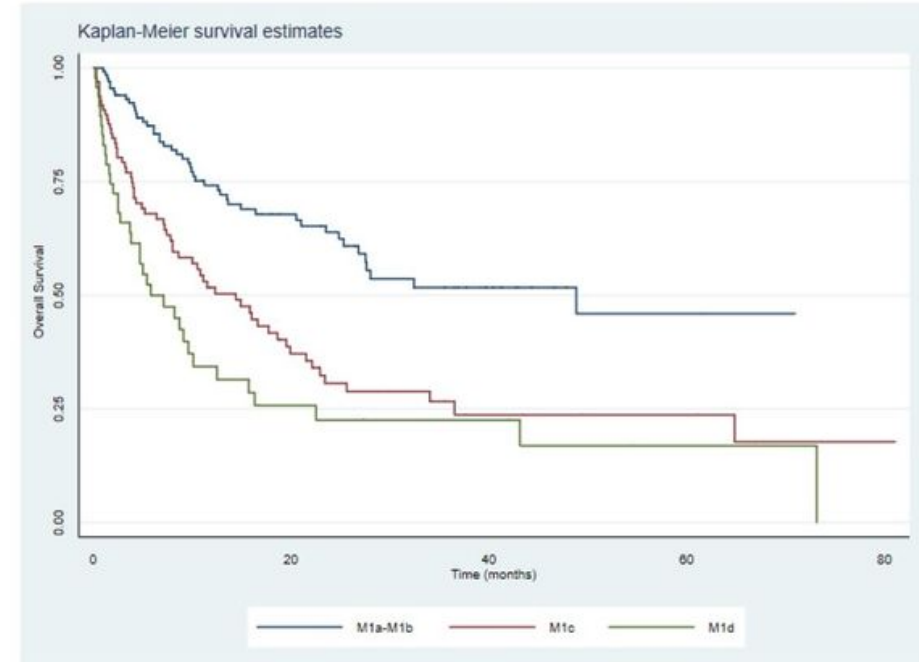

B

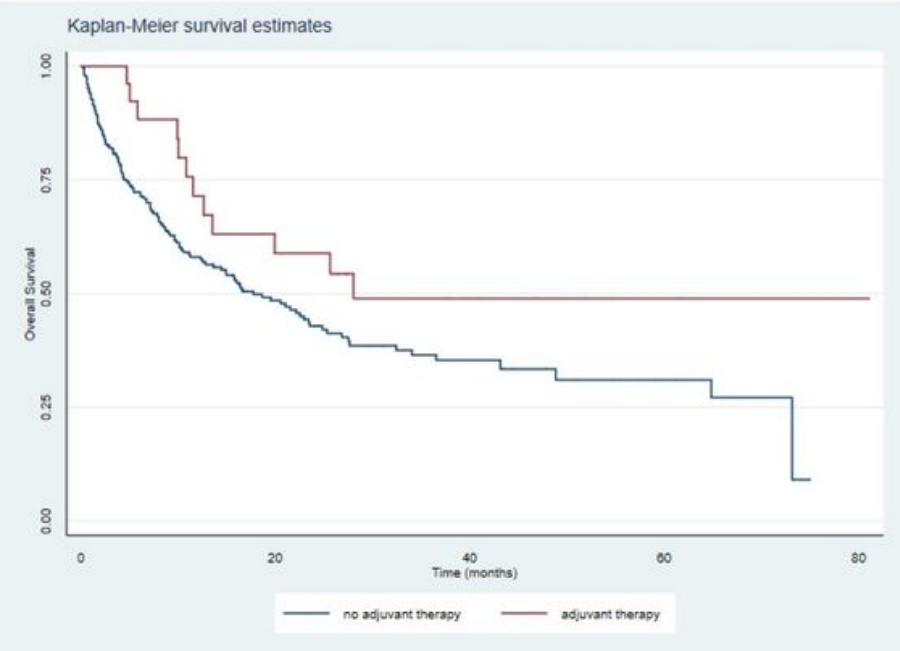

Figure 2

Overall survival analysis. Kaplan-Meier survival curves for analysis in patients with $\mathrm{M} 1 \mathrm{a} / \mathrm{b}$ or $\mathrm{M} 1 \mathrm{c}$ or M1d metastatic stage disease (A), who performed or not adjuvant treatment (B). 